\title{
Localization of lesions in endomyocardial fibrosis
}

\author{
A. G. Shaper and B. J. Bellhouse \\ From the MRC Social Medicine Unit, London School of Hygiene and Tropical Medicine; \\ and the Department of Engineering Science, University of Oxford
}

The lesions in tropical endemic endomyocardial fibrosis have a characteristic localization at the ventricular apex and on the mural endocardium behind the posterior cusp of the mitral valve. There is often a conspicuous ridge on the septal wall of the left ventricle. The use of a model left ventricle provides a possible explanation for the localization and character of the lesions in endomyocardial fibrosis. A study of ventricular diastolic flow patterns shows that the incoming jet strikes the left ventricular apex and spreads around the sides of the ventricle to produce a strong asymmetrical ring vortex. The main vortex strength and the highest wall shear stress is in the outflow tract and behind the anterior cusp. The region behind the posterior cusp is almost stagnant during diastole and the wall shear stress is very low. In systole, wall shear stress is high only in the outflow tract. The distribution of endomyocardial fibrosis appears to be confined initially to regions of low wall shear stress.

This paper concerns the mechanical factors thought to be associated with the localization of lesions in endomyocardial fibrosis; it is not concerned with aetiology, clinical manifestations, or other aspects. Endomyocardial fibrosis is a relatively common form of heart disease in many tropical countries, such as Uganda and Nigeria (Shaper, 1970). It is characterized in the established condition by fibrosis in the endocardium and subjacent myocardium of one or both ventricles, affecting particularly the inflow tract and the apex. The aetiology of this disorder is not established but it is known to be a pancarditis and in some respects its natural history is similar to that of rheumatic heart disease (Shaper, 1972). The macroscopical appearances of established endomyocardial fibrosis are distinctive and it is on these features that the necropsy diagnosis is usually made and not on isolated microscopical sections (Shaper, Hutt, and Coles, 1968).

Although endocardial lesions are the most obvious feature of the condition, there is also considerable myocardial and pericardial involvement. The inner third of the myocardium is most affected by fibrosis but it is also found throughout the width of the myocardium in all parts of the heart. The distribution resembles that seen in healed cases of myocarditis, rather than the pattern associated with ischaemia (Farrer-Brown et al., 1972). There are no abnormalities in the medium or large coronary arteries in endomyocardial fibrosis and only very Received 12 April 1973. occasionally are abnormal small vessels seen, usually within fibrous tissue areas (Farrer-Brown and Tarbit, 1972). Pericarditis is an important feature, occurring in perhaps two-thirds of the subjects with endomyocardial fibrosis and is more apparent in clinical than in pathological series of cases. A considerable body of evidence points to the generalized nature of the cardiac inflammatory response which occurs in tropical endemic endomyocardial fibrosis.

The most striking lesions in endomyocardial fibrosis occur at the ventricular apex, and on the mural endocardium behind the posterior cusp of the atrioventricular valves. Lesions may also affect both these sites with a clear area of unaffected endocardium between them. In the more acute cases of endomyocardial fibrosis, the endocardial lesions are covered with a spongy, grey-green layer of thrombus; in chronic cases the endocardial lesions are white, fibrotic, and occasionally calcified. It is generally accepted that there is a pathological sequence, starting with an acute endomyocardial lesion with overlying fibrinous deposits, to lesions with adherent thrombi and the incorporation of these thrombi into a fibrosed and contracted ventricle. The fibrosis may be localized to the apex or it may be sufficiently extensive to involve the papillary muscles and chordae tendineae, leading to atrioventricular incompetence. There is 'often a marked ridge lifted high above the adjacent endocardium on the septal wall of the left ventricle' about 2 to $3 \mathrm{~cm}$ above the apex (Davies, 1968). No such ridge 

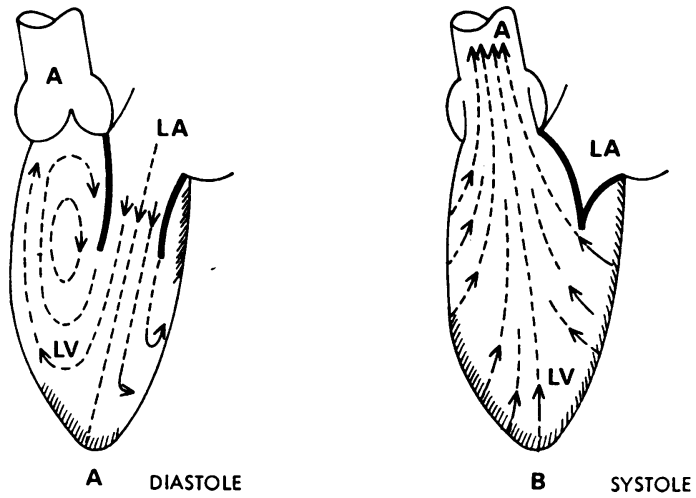

FIG. I (A) Diastolic flow patterns in the left ventricle $(L V)$. The incoming jet strikes the apex of the ventricle to form a strong ring vortex, with its main strength concentrated in the outflow tract. The wall shear stress is low in the (shaded) regions near the apex and behind the posterior cusp of the mitral valve. (B) Systolic flow patterns in the left ventricle $(L V)$. The region of low wall shear stress (shaded) extends from the apex to behind the posterior cusp. $L A$, left atrium; $A$, aorta.

is seen in the right ventricle, though right ventricular endomyocardial fibrosis can be very severe and produce almost complete obliteration of the cavity.

How can one explain the localization of the lesions in endomyocardial fibrosis ? It could simply be that the disease process, for reasons unknown, is localized to certain very specific areas, in the way that rheumatic heart disease shows an unexplained predilection for the heart valves. However, it is more likely that the disorder is initially widespread in the heart, possibly involving most of the endocardium in the acute stage, and that for certain haemo- dynamic reasons, fibrin deposition, thrombus formation, organization, and scarring take place in the characteristic sites. The use of a model left ventricle developed by Bellhouse (1972) provides a possible explanation for the localization and character of the lesions in endomyocardial fibrosis, in terms of the distribution of wall shear stress, the frictional drag per unit area on the ventricular wall produced by blood flowing over it.

It has been shown that the mitral valve opens rapidly at the beginning of diastole and the incoming jet strikes the apex of the ventricle (Fig. IA). It then spreads outwards and upwards around the sides of the ventricle to produce a strong asymmetrical ring vortex, with its main strength concentrated in the outflow tract. Where the jet strikes the apex of the ventricle, in an area roughly equal in diameter to the mitral annulus, the flow is brought to rest, and the shear stress developed between the blood and the ventricular wall is low. Away from the apex, blood velocity near the wall increases and wall shear stress rises. Since the main vortex strength is concentrated in the outflow tract, this region is scoured in diastole, in contrast to the region behind the posterior cusp, which is almost stagnant in diastole.

In systole the streamlines are very different (Fig. IB). The streamlines originate at the wall, and near the apex are almost perpendicular to it. Wall shear stress is negligible in this apical region. In the outflow tract the streamlines bend towards the aorta, and blood velocities, and hence wall shear stresses, are high.

Thus both in systole and diastole wall shear stress is high in the outflow tract and behind the anterior cusp of the mitral valve. Regions behind the posterior cusp and near the apex encounter low wall shear stress in both systole and diastole. A ring, surrounding a localized area at the apex, experiences

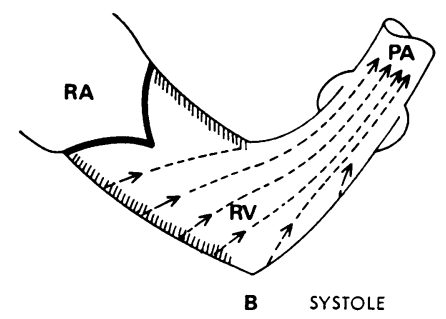

FI G. 2 (A) Diastolic flow patterns in the right ventricle ( $R V)$. The incoming jet will strike the ventricle near the apex to form a vortex. Regions of low wall shear stress will exist near the apex and behind some of the cusps of the tricuspid valve. $(B)$ Systolic flow patterns in the right ventricle $(R V)$. The region of low wall shear stress is mainly behind the cusps of the tricuspid valve, and may not extend as far as the apex of the right ventricle. $R A$, right atrium; $P A$, pulmonary artery. 
high wall shear stress in diastole and low wall shear stress in systole.

If the endocardial lesion in endomyocardial fibrosis is initially diffuse, then the area where a return to normality is least likely to take place is in a region of low shear stress. When an endomyocardial lesion is established, fibrin deposition and thrombus incorporation could proceed undisturbed in these regions.

These regions are the apex of the left ventricle and the area behind the posterior cusp of the mitral valve, with an unaffected area separating the two, the sites which are indeed affected in endomyocardial fibrosis. From model experiments (Bellhouse, 1972) we estimate that wall shear stress can reach about 40 dynes $/ \mathrm{cm}^{2}$ in diastole in regions of high shear and zero in regions of low shear. This effect cannot easily be dismissed as insignificant when compared with Fry's (1968) experiments. He showed that trauma of normal endothelial cells occurred when wall shear stresses reached about 380 dynes $/ \mathrm{cm}^{2}$.

An alternative explanation to the one proposed in this paper is that the apical lesion is due to impact of the jet in diastole or atrial systole. The jet has a dynamic head of only I $\mathrm{mmHg}$ (when its velocity is $50 \mathrm{~cm} / \mathrm{sec}$ ). So this explanation seems improbable though unsteady effects may be greater. This explanation would not, of course, apply to the other lesions in the left ventricle.

Although comparable studies have not been reported for the right ventricle, it is probable that a diastolic vortex is established in the right ventricle, which aids tricuspid valve closure in much the same way as occurs in the left ventricle (Fig. 2A). However, the shape of the right ventricle is such that wall shear stress at the apex of the right ventricle, though low in diastole, will be relatively high in systole (Fig. 2B). This could explain why no ridge is found surrounding the apical region of the right ventricle.

\section{References}

Bellhouse, B. J. (1972). Fluid mechanics of a model mitral valve and left ventricle. Cardiovascular Research, 6, 199.

Davies, J. N. P. (1968). The ridge in endomyocardial fibrosis. Lancet I, 631 .

Farrer-Brown, G., and Tarbit, M. H. (1972). Heart involvement in endomyocardial fibrosis. British Heart fournal, 34, I062.

Farrer-Brown, G., Tarbit, M. H., Somers, K., and Hutt, M. S. R. (1972). Microvascular study of hearts with endomyocardial fibrosis. British Heart fournal, 34, 1250.

Fry, D. L. (1968). Acute vascular endothelial changes associated with increased blood velocity gradients. Circulation Research, 22, 165 .

Shaper, A. G. (1970). The geographical distribution of endomyocardial fibrosis. Pathologia et Microbiologia, 35, 26.

Shaper, A. G. (1972). Cardiovascular disease in the tropics. II. Endomyocardial fibrosis. British Medical fournal, 3, 743.

Shaper, A. G., Hutt, M. S. R. and Coles, R. M. (1968). Necropsy study of endomyocardial fibrosis and rheumatic heart disease in Uganda 1950-1965. British Heart fournal, 30, 39r.

Requests for reprints to Dr. A. G. Shaper, MRC Social Medicine Unit, London School of Hygiene and Tropical Medicine, Keppel Street (Gower Street), London WCIE $7 \mathrm{HT}$. 